\title{
La resoluciones de problemas a través de mapas conceptuales móviles cooperativos.
}

\section{Virginia Santamarina Campos ${ }^{\mathrm{a}}$, María Ángeles Carabal Montagud ${ }^{\mathrm{b}}$, María de Miguel Molina $^{c}$ y Blanca de Miguel Molina ${ }^{d}$}

\author{
${ }^{a}$ Departamento de Conservación y Restauración de Bienes Culturales, Universitat Politècnica de \\ València (UPV) virsanca@upv.es \\ ${ }^{b}$ Departamento de Conservación y Restauración de Bienes Culturales, Universitat Politècnica de \\ València (UPV) macamon@crbc.upv.es \\ ${ }^{c}$ Departamento de Organización de Empresas, UPV mademi@omp.upv.es \\ ${ }^{\mathrm{d}}$ Departamento de Organización de Empresas, UPV bdemigu@omp.upv.es
}

\begin{abstract}
El aprendizaje cooperativo, basado en la resolución de problemas, mediante el diseño de mapas conceptuales móviles, proporciona claridad, ayudando a hacer las ideas visibles, tangibles y secuenciales. Las tarjetas móviles, permiten obtener modelos de sistemas más eficaces, al permitir la reiteración con mayor fluidez. Por otro lado el trabajo en grupo con el empleo de notas móviles da como resultado un modelo sistémico más rico, porque sintetiza diferentes puntos de vista, fusionando modelos mentales, y facilitando la tarea de entender y resolver problemas complejos de forma cooperativa, creativa e innovadora.
\end{abstract}

Keywords: aprendizaje cooperativo, innovación docente, trabajo en grupo, mapas conceptuales, mapas mentales, modelos mentales, tarjetas móviles, análisis de problemas, resolución de problemas, diseño de proyectos.

\begin{abstract}
Cooperative learning, based on problem solving and the design of conceptual mobile maps, provides clarity and helps to make ideas more visible, tangible and sequential. Mobile cards allow to obtain system models more efficient by allowing more smoothly reiteration. On the other hand, teamwork with the use of mobile notes results in a richer systemic model, because it synthesizes different viewpoints, merging mental models and making it easier to understand and solve complex problems in a cooperative, creative and innovative way.
\end{abstract}


Keywords: cooperative learning, teaching innovation, teamwork, conceptual maps, mind maps, mental models, mobile cards, problem analysis, problem solving, project design

\section{Introducción}

La nueva concepción del patrimonio cultural, exige replantear una importante reflexión sobre los nuevos modelos de actuación patrimoniales. El turismo, la globalización y la patrimonialización de nuevos elementos culturales, exigen la revisión y propuesta de nuevos métodos de intervención, en los que se contemplen los aspectos socioculturales, sus valores de uso, formal y simbólico/identitario.

Este cambio en la percepción y manejo del patrimonio cultural, ha supuesto una transformación profunda en el diseño de proyectos de conservación y restauración. Este hecho abre camino a la aceptación de una participación intersubjetiva y a una consideración de la disciplina más amplia, que transcienda los límites del estudio objetual y recopilatorio, y conceda mayor protagonismo a los sujetos receptores y los diferentes procesos de significación, concibiéndose esta actividad profesional a partir de "sus usos sociales, no desde una mera actitud defensiva, de simple rescate, sino con una visión más compleja de cómo la sociedad se apropia de su historia, puede involucrar a nuevos sectores. No tiene porqué reducirse a un asunto de los especialistas del pasado [...]" (García Canclini, 1993).

En relación con lo anterior, pasar del objeto de restauración al sujeto que le otorga un conjunto de valores, como motivo de estudio implica la necesidad de plantear, desde el análisis y la reflexión, un proceso de deconstrucción, reinterpretación y redefinición de criterios anteriores sólidamente asentados en el campo de la conservación y restauración de bienes culturales (tales como la objetividad, autenticidad, verdad, materialidad,...) por otros que abren camino a la vía de la subjetividad.

Esto supone la necesidad de descubrir en el alumno una postura definida, crítica y creativa frente a la importancia de la construcción social del patrimonio, para lograr la gestión sostenible del patrimonio cultural. En el diseño de proyectos, por tanto, la fase más importante será la de identificación, en la que se analizará la situación existente para crear una visión de la situación deseada y seleccionar las estrategias que se emplearán para lograrla. Hasta ahora la herramienta empleada en el análisis de problemas para poder plantear una propuesta de intervención patrimonial, era el método dafo. Este método permite identificar los puntos fuertes y débiles de un área concreta y las amenazas y oportunidades del entorno, obteniendo una imagen global de la problemática del recurso patrimonial. A pesar de que esta técnica puede ayudar en la elaboración de estrategias o alternativas que mitiguen las debilidades a partir de las potencialidades y fortalezas existentes, no permite obtener una visión objetiva del problema focal, así como de sus 
causas y efectos. Actualmente la visualización de las relaciones causales de los problemas de una forma clara, supone una tarea fundamental ya que no solo nos importa el objeto, sino la relación con de los sujetos que le otorgan un conjunto de valores.

Por tanto la elaboración de un diagnostico que permita obtener un conocimiento profundo de la situación como base para la toma de decisiones, exige el empleo de una herramienta que permita entender porque se ha producido esa situación no desea y cuales con sus consecuencias, facilitando la priorización de los problemas y el llegar a un acuerdo colaborativo en el diagnóstico entre implicados.

Por este motivo en los ultimo años el análisis de problemas se dirige a establecer las relaciones causa-efecto entre los problemas existentes (Fig. 1), que han sido identificados previamente por diversos actores (Ferrero y de Loma-Osorio, 2009). En este caso los mapas conceptuales móviles cooperativos, han permitido visualizar las relaciones causales, priorizar los problemas y llegar a un acuerdo en el diagnóstico entre implicados, a través del trabajo colaborativo. La posibilidad de trabajar con mapas móviles, ha permitido la revisión colaborativa hasta verificar que el sistema es valido, haciendo los ajustes necesarios y llegando a consenso sobre su estructura.

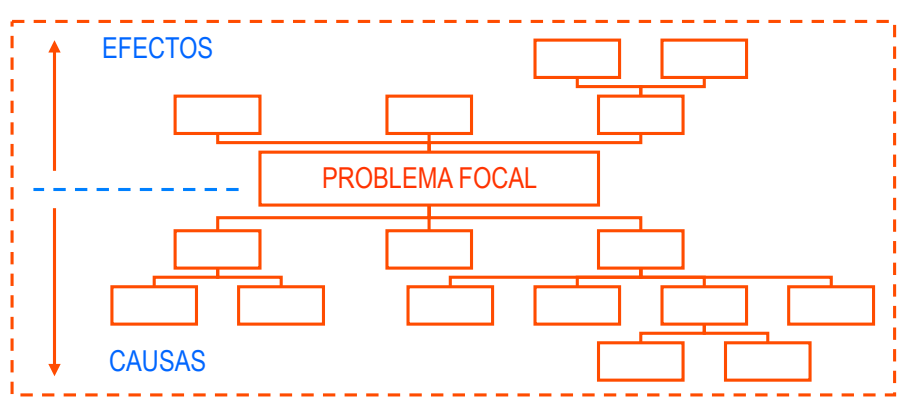

Fig. 1 Análisis de problemas.

\section{Objetivos}

El objetivo principal de esta propuesta, es fomentar el empleo de mapas conceptuales móviles cooperativos, para la elaboración del análisis de problemas en el ámbito del patrimonio cultural, que les permita obtener modelos de sistemas más eficaces, al permitir la reiteración con mayor fluidez, y modelos sistémicos más eficientes a través del trabajo el grupo, al sintetizar diferentes puntos de vista.

Por tanto el objetivo final, será ser capaces de entender y resolver problemas complejos en el ámbito de la gestión del patrimonio cultural, para responder a sus necesidades, de forma cooperativa, dinámica, creativa e innovadora. 


\section{Desarrollo de la innovación}

A pesar de que el empleo de los mapas conceptuales móviles se utilizan en el aula desde el 2005 (Fig.2 y 3), en los dos últimos años, se ha incorporado una nueva práctica, para que el propio alumno, reflexione y sea consciente de las ventajas que aporta el uso de tarjetas móviles de forma cooperativa para la comprensión y resolución de problemas complejos.
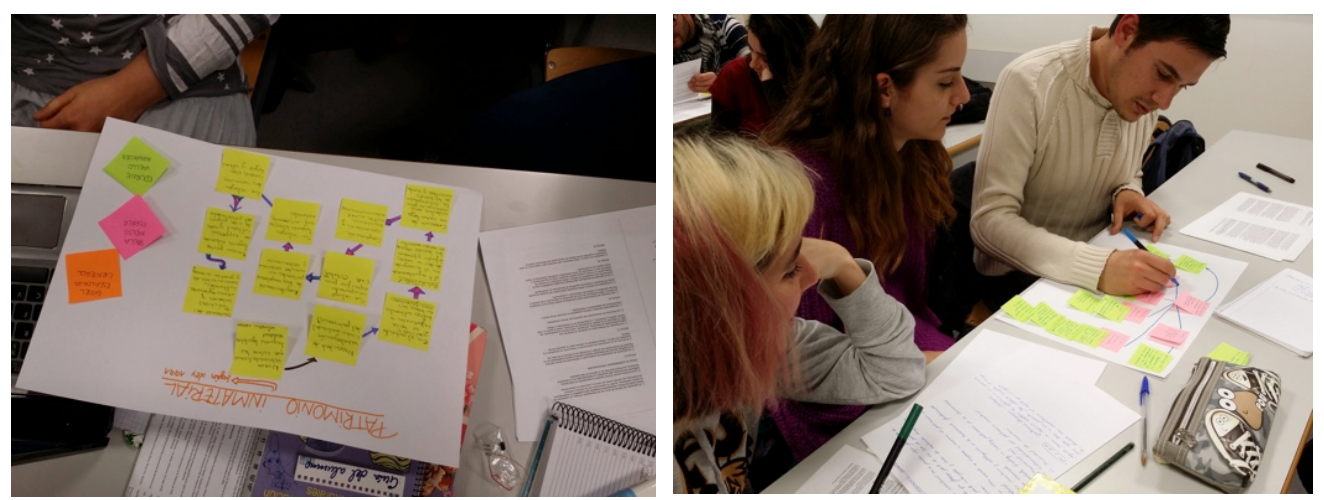

Fig. 2 y 3 Empleo de mapas conceptuales móviles. Máster Universitario en Conservación y Restauración de Bienes Culturales, del Departamento de Conservación y Restauración de Bienes Culturales de la UPV. Curso 2014-2015.

Para ello se les plantea un sencillo ejercicio de diseño que ayuda a los alumnos a entender y resolver problemas complejos (Wujec, 2013), dividido en tres partes secuenciales:

\section{Descripción de la dinámica:}

\section{$1^{a}$ parte}

Modo trabajo: individual.

Material: hoja de papel A3 y un marcador.

Descripción: Sin utilizar palabras, se les indica que dibujen un proceso de restauración basándose en la patología que visualizan en una imagen (Fig. 4 y 5). Se busca representar un proceso de intervención sencillo que resulte familiar a cualquier alumno, a pesar de haber realizado especialidades diferentes. Tras finalizar los trabajos, se pegan en la pared y todo el grupo analiza qué cualidades tienen en común. Se lanzan las siguientes preguntas y se debate en grupo:
$1^{\circ} \quad$ ¿Qué tienen en común los diseños?
$2^{\circ} \quad$ ¿Qué elementos contienen los diseños?
$3^{\circ} \quad$ ¿Qué representan los nodos?
$4^{\circ} \quad$ ¿Qué representan los enlaces? 


\section{Resultados:}

\section{$1^{0}$ ¿Qué tienen en común los diseños?}

El 90\% de los diseños siempre presentan nodos y enlaces.

\section{$2^{\circ}$ ¿Qué elementos contienen los diseños?}

Los diseños combinan enlaces y nodos, dando lugar a un modelo de sistema completo, que hace visible nuestros modelos mentales privados sobre cómo pensamos que funciona algo, convirtiéndose en "una poderosa técnica gráfica que nos ofrece una llave maestra para acceder al potencial del cerebro" (Buzán, 1996).

Cada diseño muestra un nivel de complejidad en función del número de nodos que suelen ir entre 6 y 8 . Los que utilizan menos nodos son más sencillos de entender, pero parece triviales, mientras que los que emplean más de 13 dan como resultado mapas complejos que dificultan su comprensión. Por lo que el número adecuado de nodos en un mapa, si se quiere mostrar algo de forma visual, debería ser entre 5 y 13.

\section{$3^{\circ}$ ¿Qué representan los nodos?}

Los nodos representan los objetos tangibles, como las herramientas y materiales utilizadas para el proceso de restauración, el restaurador, la obra etc.

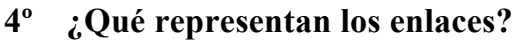

Los enlaces representan las conexiones entre los nodos, dando lugar a mapas conceptuales que hacen visible nuestro modelos mentales.

\section{Conclusión:}

Los diferentes diseños, representan mapas conceptuales, que revelan puntos de vista diferentes (Fig. 5 y 6). A pesar de no ser expertos en diseño de mapas conceptuales, logran de forma intuitiva dividir procesos complejos en tareas simples, organizándolo y sintetizarlo en un mapa.

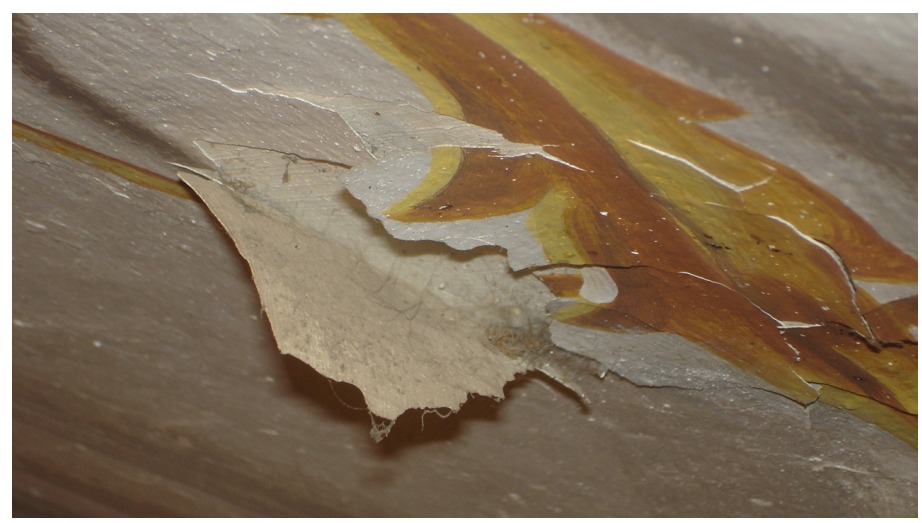

Fig. 4. $1^{a}$ Etapa del ejercicio. Imagen de la patología que deben resolver en la $1^{a}$ etapa del ejercicio. Asignatura Conservación preventiva, curso 2015-2016. Grado en Conservación y Restauración de Bienes Culturales, del Departamento de Conservación y Restauración de Bienes Culturales de la UPV.

(c)) EY-NC-ND 2016, Universitat Politècnica de València 


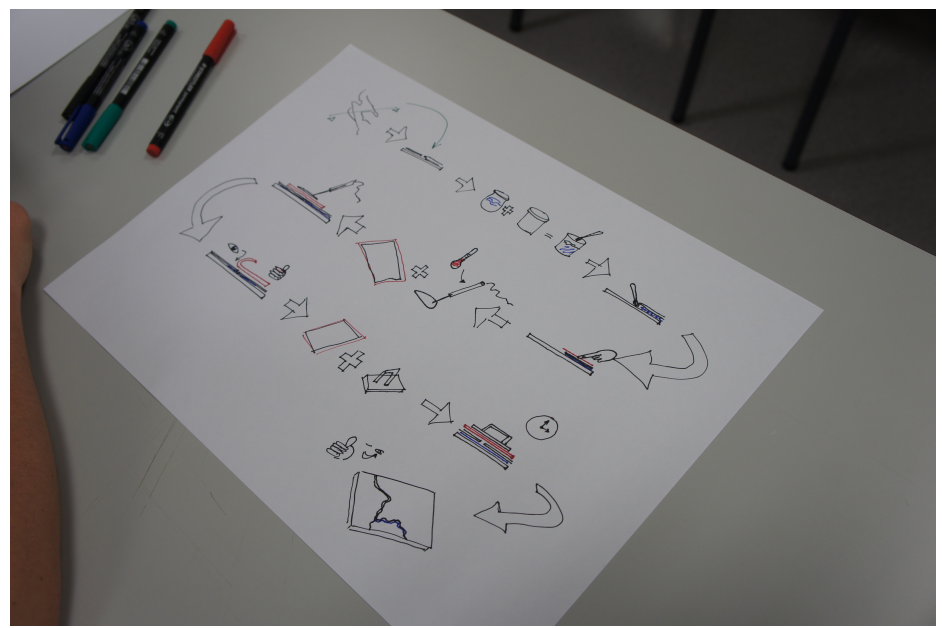

Fig. 5. $1^{a}$ Etapa del ejercicio. Detalle de uno de los mapas conceptuales elaborados en la $1^{a}$ etapa del ejercicio. Asignatura Conservación preventiva, curso 2015-2016. Grado en Conservación y Restauración de Bienes Culturales, del Departamento de Conservación y Restauración de Bienes Culturales de la UPV.
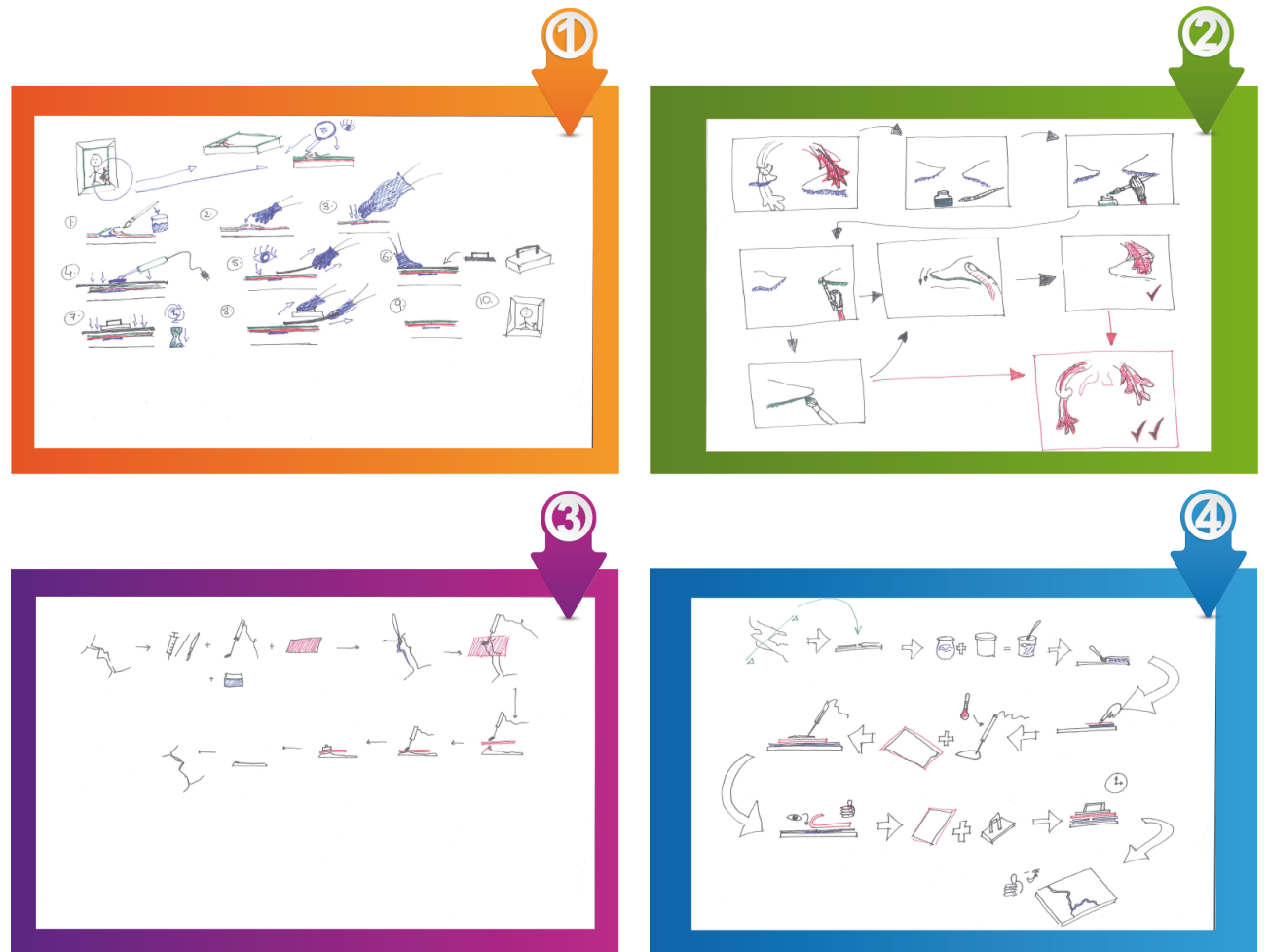

Fig. 6. $1^{a}$ Etapa del ejercicio. Resultado de los 4 mapas conceptuales realizados de forma individual en la $1^{a}$ etapa del ejercicio, por alumnos que después trabajaran en grupo. Asignatura Conservación preventiva, curso 2015 2016. Grado en Conservación y Restauración de Bienes Culturales, del Departamento de Conservación y Restauración de Bienes Culturales de la UPV.

(cc) EY-NG-ND 2016, Universitat Politècnica de València 


\section{$2^{a}$ parte}

Modo trabajo: individual.

Material: hoja de papel A3, un marcador y post-it.

Descripción: Sin utilizar palabras, dibujar el proceso descrito en el ejercicio anterior, pero esta vez utilizando tarjetas móviles de colores, un dibujo por post-it, y los post-it se van colocando y organizando en una hoja de formato A3 (Fig.7). Tras finalizar los trabajos, se forman grupos de entre 3 y 5 alumnos y se analiza qué cualidades tienen en común (Fig. 8 y 9). Se lanzan las siguientes preguntas y se debate en grupo:

$1^{\mathrm{o}}$ ¿Qué tienen en común los diseños?.

$2^{\circ} \quad ¿ Q u e ́$ diferencia hay entre el $1^{\circ}$ ejercicio y el $2^{\circ}$ ? (Fig.10).

\section{Resultados:}

\section{$1^{\circ}$ ¿Qué tienen en común los diseños?}

La mayor parte de los alumnos, tiende a dibujar nodos claramente más detallados y lógicos. Los mapas son mucho más ricos en detalles.

\section{$2^{\circ}$ ¿Qué diferencia hay entre el $1^{\circ}$ ejercicio y el $2^{\circ}$ ?}

Pueden ver paso a paso el análisis que se lleva a cabo, y mientras construyen su mapa, pueden mover los nodos reorganizándolos.

\section{Conclusión:}

La facilidad con la que se puede modificar un mapa, está vinculada con nuestro interés de mejorar el modelo. Así que un sistema con notas post-it no solo es más dinámico y flexible sino que también genera más nodos que los dibujos estáticos.

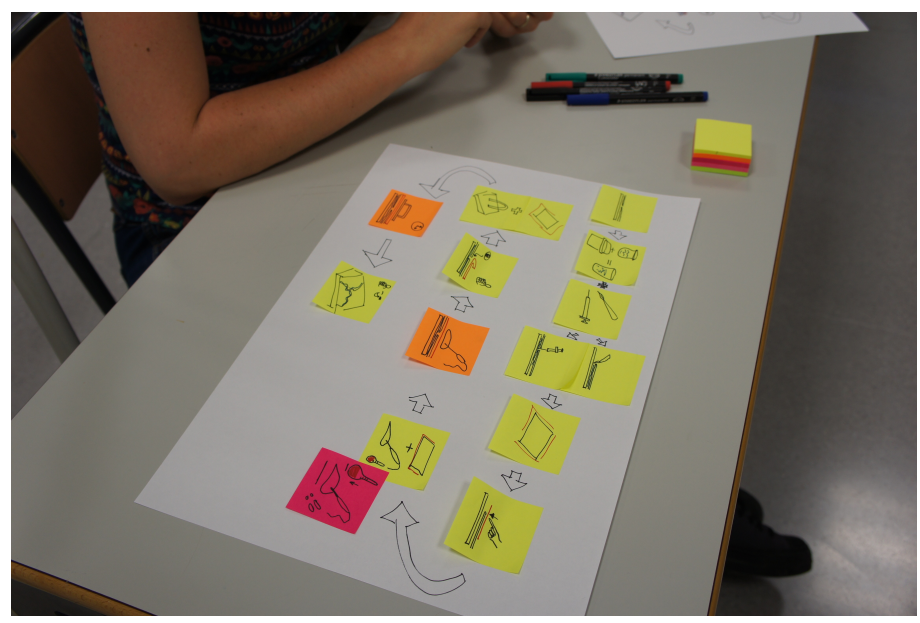

Fig. 7. $2^{a}$ Etapa del ejercicio. Sin utilizar palabras, de forma individual, dibujar el proceso en tarjetas móviles. Posteriormente realizar un análisis de las cualidades que tiene en común los ejercicios de la $1^{a}$ y $2^{a}$ etapa. 
Asignatura Conservación preventiva, curso 2015-2016. Grado en Conservación y Restauración de Bienes Culturales, del Departamento de Conservación y Restauración de Bienes Culturales de la UPV.

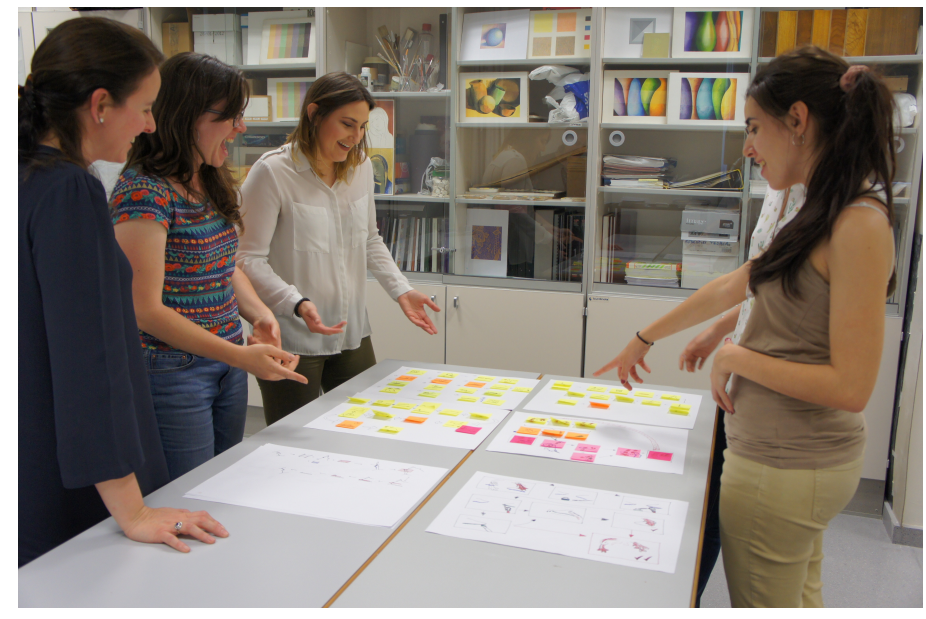

Fig. 8. $2^{a}$ Etapa del ejercicio. Puesta en común y análisis de los mapas. Asignatura Conservación preventiva, curso 2015-2016. Grado en Conservación y Restauración de Bienes Culturales, del Departamento de Conservación y Restauración de Bienes Culturales de la UPV.

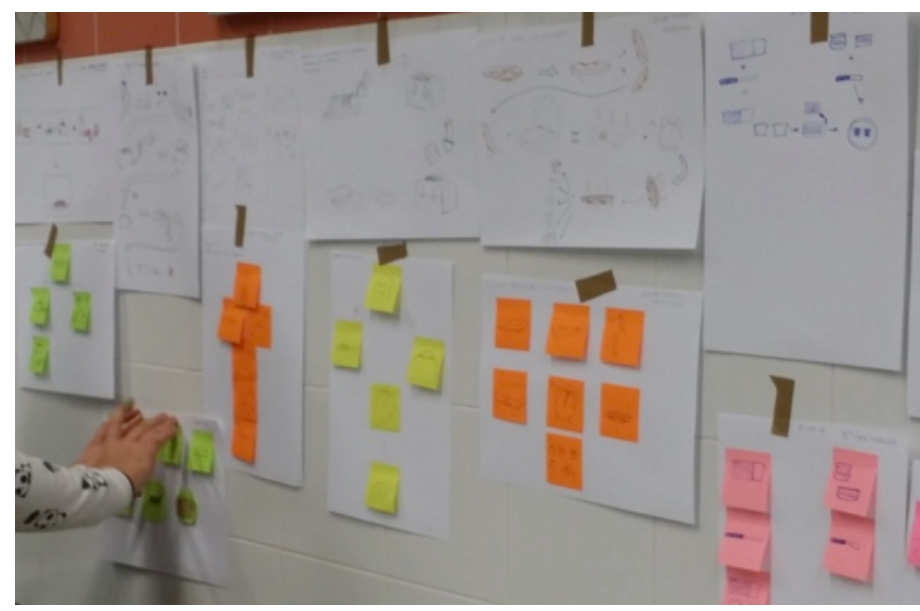

Fig.9. $2^{a}$ Etapa del ejercicio. Análisis de los mapas. Asignatura Desarrollo, gestión y dirección de proyectos de restauración, curso 2015-2016. Máster Universitario en Conservación y Restauración de Bienes Culturales, del Departamento de Conservación y Restauración de Bienes Culturales de la UPV. 


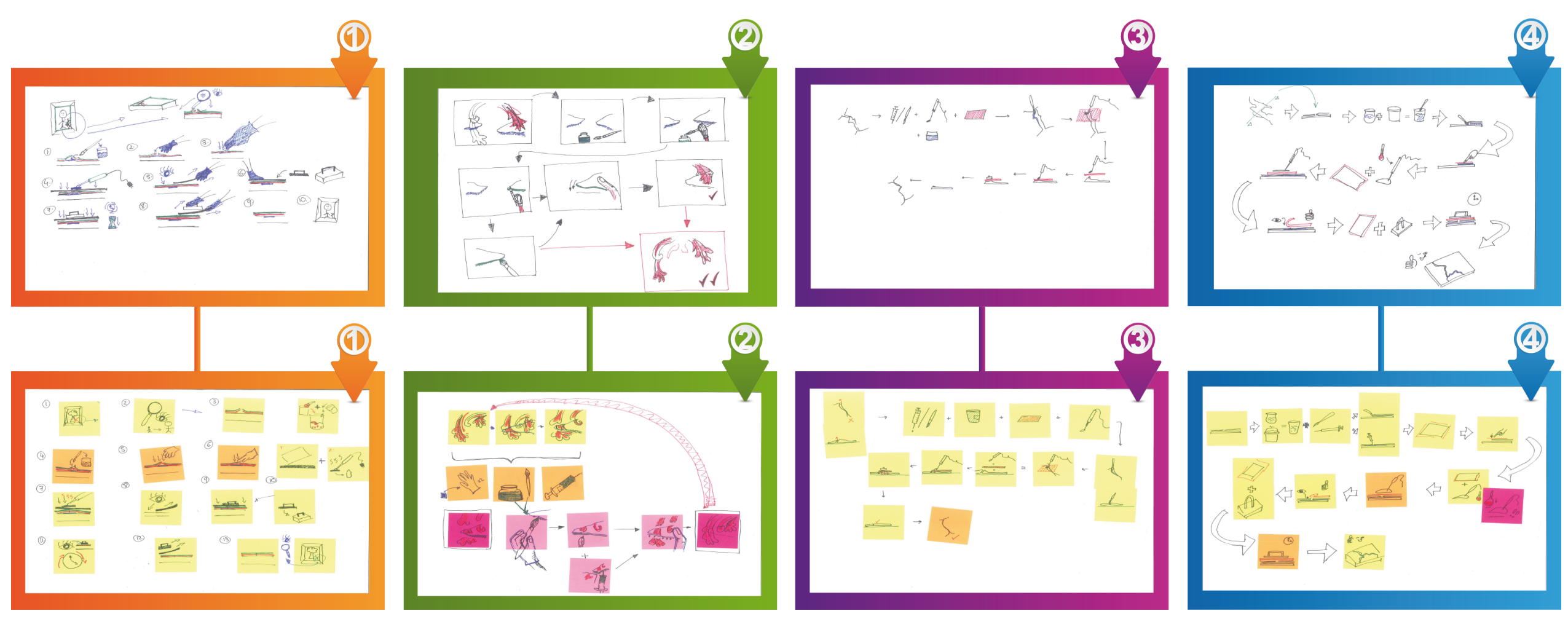

Fig. 10. $2^{a}$ Etapa del ejercicio. Sin utilizar palabras, de forma individual, dibujar el proceso en tarjetas móviles. Posteriormente realizar un análisis de las cualidades que tiene en común los ejercicios de la $1^{a}$ y $2^{a}$ etapa. Asignatura Conservación preventiva, curso 2015-2016. Grado en Conservación y Restauración de Bienes Culturales, del Departamento de Conservación y Restauración de Bienes Culturales de la UPV. 


\section{$3^{a}$ parte}

Modo trabajo: grupal.

Género: paridad

Número: mínimo 3, máximo 5.

Material: hoja de papel A3, un marcador y post-it de colores.

Descripción: Partiendo de los mapas realizados en el ejercicio anterior, se repite el proceso reutilizando las tarjetas, pero esta vez se trabaja en grupo (Fig.11). Tras finalizar los trabajos, cada grupo analiza su mapa resultante (Fig. 12), y para concluir toda la clase analiza qué cualidades tienen en común los mapas generados por cada grupo (Fig.11). Se lanzan las siguientes preguntas y se debate en grupo:

$1^{\circ} \quad$ ¿Qué diferencia hay entre el $2^{\circ}$ ejercicio y el $3^{\circ}$ ?

\section{Resultados:}

Parten de los modelos desarrollados de forma individual, perfeccionan sus mapas y los mejores nodos se vuelven más visibles, y con cada repetición, el modelo se vuelve más claro porque los alumnos se basan en las ideas de los demás.

\section{Conclusión:}

Tenemos como resultado, mapas conceptuales unificados que integran la diversidad de puntos de vista individuales de todos los alumnos. Estos nuevos sistemas contienen un elevado número de nodos, incluso nuevas ramificaciones y patrones, empleando diferentes colores de tarjetas, pero para los alumnos no resultan complejos porque participan en la construcción de estos nuevos modelos comunes.

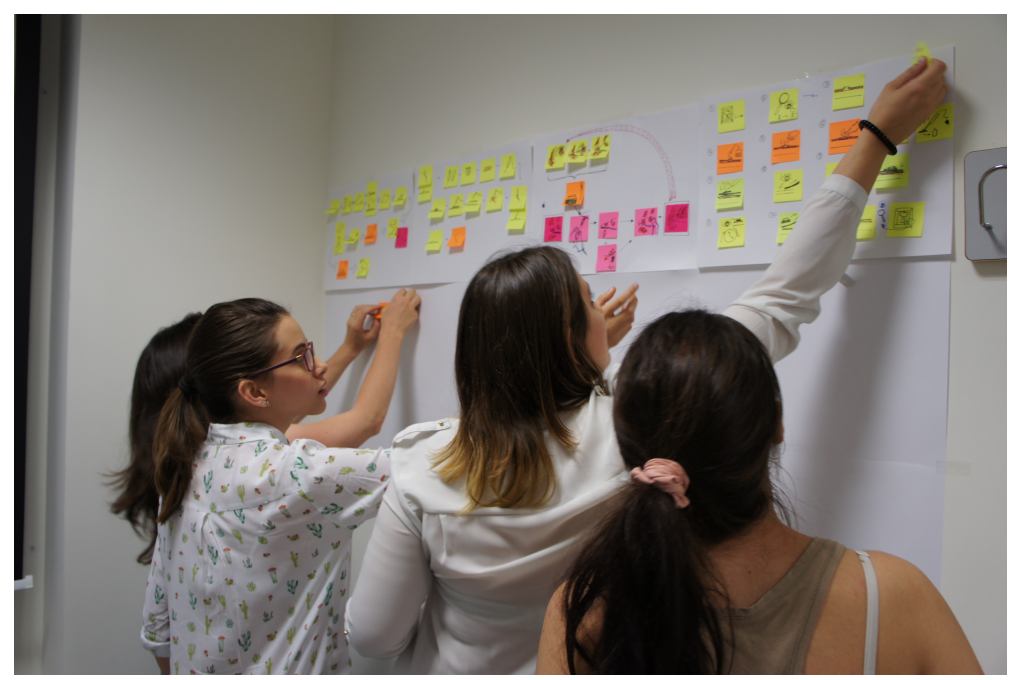

Fig. 11. 3a Etapa del ejercicio. Partiendo de los mapas realizados en el ejercicio anterior, se repite el proceso reutilizando las tarjetas, pero esta vez se trabaja en grupo. Asignatura Conservación preventiva, curso 2015- 


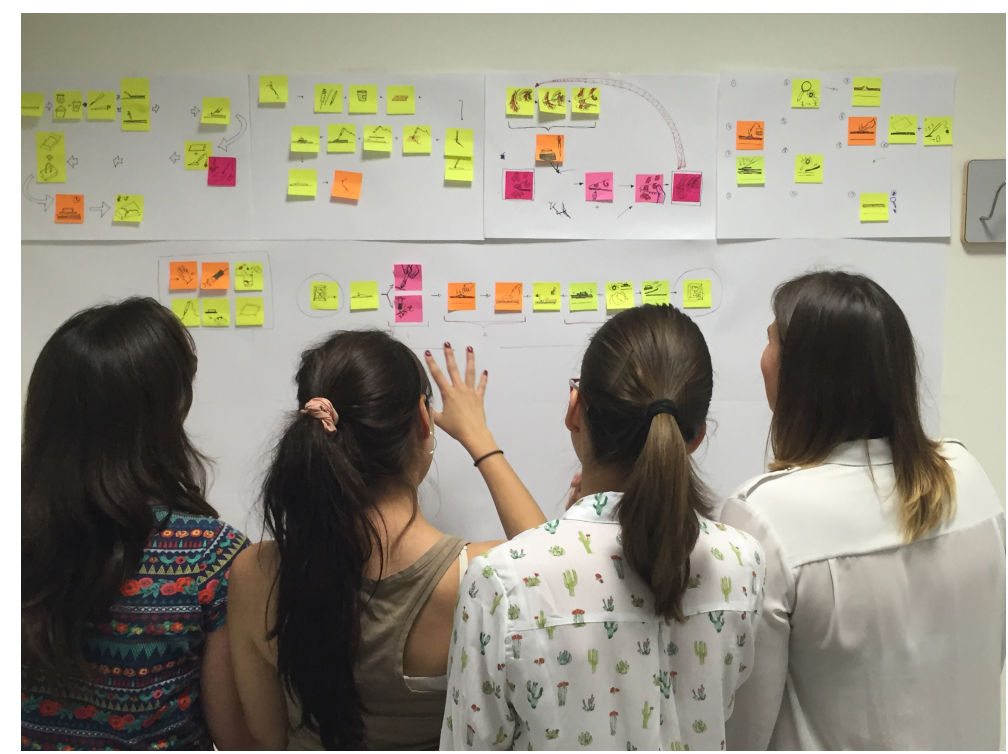

Fig. 12. $3^{a}$ Etapa del ejercicio. Para concluir se analiza el mapa colaborativo resultante. Asignatura Conservación preventiva, curso 2015-2016. Grado en Conservación y Restauración de Bienes Culturales, del Departamento de Conservación y Restauración de Bienes Culturales de la UPV.

\section{Resultados}

El proceso de abordar el diseño de mapas conceptuales para resolver problemas, les permite descubrir que dibujar o emplear palabras claves les ayuda a hacer sus ideas visibles, tangibles y secuenciales, y por tanto, a entender los problemas como sistemas de nodos y relaciones. Por otro lado el trabajo con tarjetas móviles (post-it) les ayuda fomentar el pensamiento divergente, a mejorar la organización y estructuración de los mapas, porque la reiteración se hace con mayor fluidez. El empleo de tarjetas móviles de colores también les permite una mejor estructuración de los mapas, atribuyendo el color de las tarjetas a categorías, características de los procesos, etc., como se puede observar en la figura 13, donde para los procesos que desprenden calor, en todos los casos han utilizado el naranja intenso, mientras que para procesos delicados donde se precisa mucho control, emplean tarjetas rosas. Por último el trabajo en grupo con notas móviles produce el mejor modelo sistémico, porque sintetiza diferentes puntos de vista, enriqueciendo los mapas conceptuales, y ayudando a resolver problemas complejos a través de la visualización colaborativa (Fig 13). En opinión de los alumnos, la última etapa de la dinámica, siempre resulta la más satisfactoria, enriquecedora y clarificante, algunos de los comentarios que hemos podido rescatar de las sesiones que han sido filmadas, en relación a la $3^{\text {a }}$ etapa del ejercicio son: "Este esta más completo", "Esta más claro", y "El poner el común lo que hemos hecho, ha hecho que podamos construir un proceso en el que a lo mejor se nos había olvidado algo, y otra persona si que lo había puesto". 

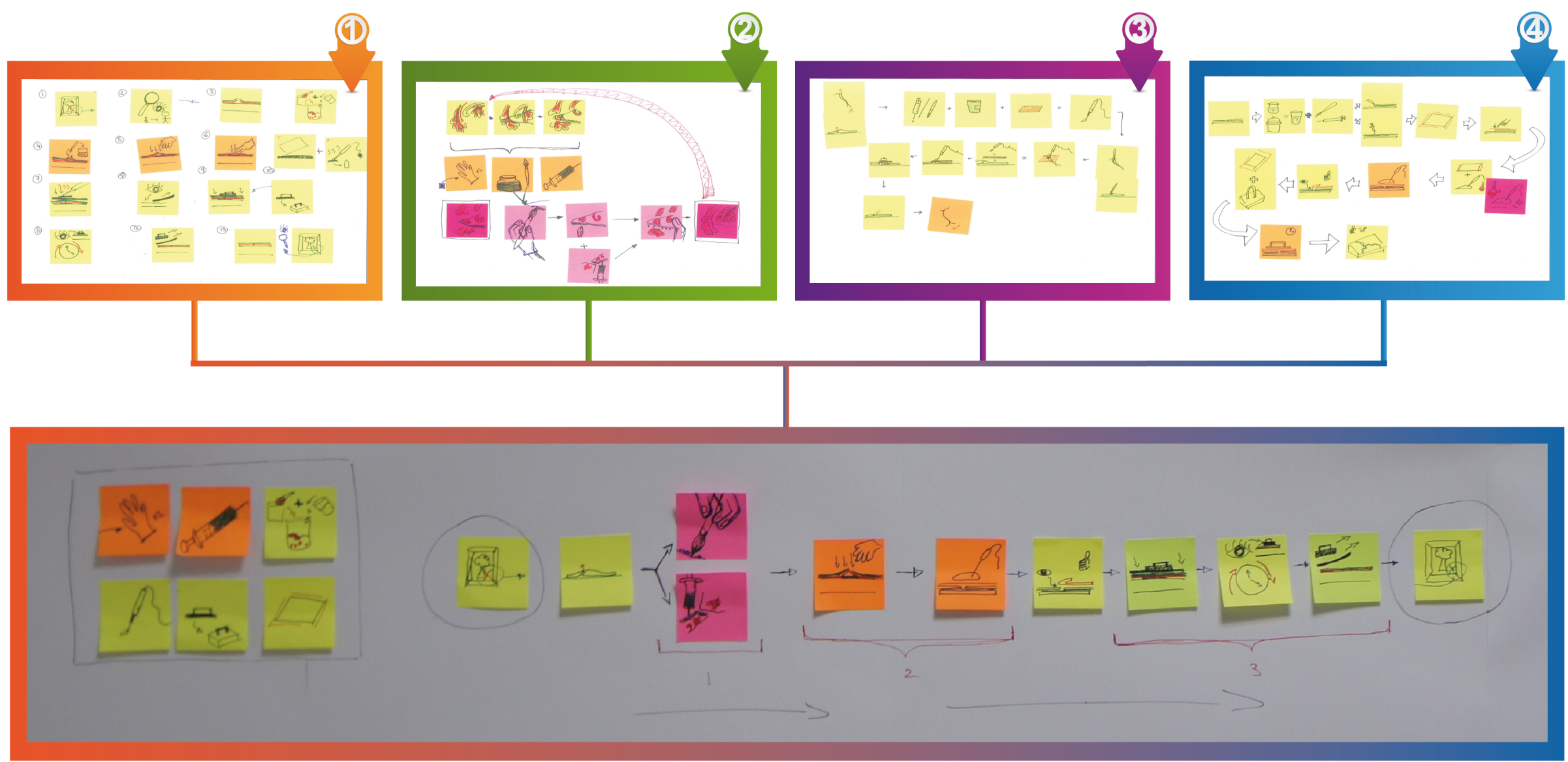

\section{$\mathrm{O}+\mathrm{O}+\mathrm{O}+\mathrm{O}$}

Fig. 13. Sintesis del proceso de la $3^{a}$ Etapa del ejercicio. Asignatura Conservación preventiva, curso 2015-2016. Grado en Conservación y Restauración de Bienes Culturales, del Departamento de Conservación y Restauración de Bienes Culturales de la UPV. 


\section{Conclusiones}

La transformación del patrimonio, ha supuesto un nuevo enfoque en su gestión e intervención, que hace necesaria la incorporar de aspectos relacionados con la dimensión simbólica-identitaria, política y económica en los procesos de análisis, para comprender el problema focal, así como las causas y efectos que derivan de éste. El proceso de análisis colaborativo del problema, supone un paso fundamental en el correcto diseño del proyecto, dado que ayuda a visualizar las relaciones causales entre los mismos y el diagnóstico entre implicados. Abordar esta tarea sin el apoyo de un mapa conceptual móvil cooperativo, supone un trabajo complejo, que puede dar lugar a múltiples errores que después serán arrastrados durante el diseño del proyecto.

El empleo de este ejercicio en el inicio de las materias en las que tienen que abordar el diseño de proyectos, les permite descubrir y ser conscientes, a través de un ejercicio sencillo, de las ventajas que plantea este método de trabajo, para la resolución eficaz de problemas complejos, reflejando el pensamiento de todos los componentes del grupo y potenciando el desarrollo de relaciones interpersonales positivas entre los mismos (Muñoz González, 2014). De este modo el uso de mapas conceptuales móviles cooperativos, se emplea como estrategia de aprendizaje y al mismo tiempo sirven como herramienta básica para compartir los conocimientos en la resolución de problemas.

El alcance de este método de trabajo, queda patente en las exposiciones realizadas por los alumnos de trabajos finales, tesis de grado, master y doctorado, que se presentan en el Departamento de Conservación y Restauración de Bienes Culturales de la UPV (Fig.14 y 15), en las que de forma sistemática incorporan mapas conceptuales, para la visualización de los problemas a resolver. Por otro lado el nivel de satisfacción de los alumnos con el empleo de esta técnica queda patente en los resultados de las encuestas, en las que tras la implementación de esta herramienta aumentan entre un 10 y $15 \%$ la valoración final.
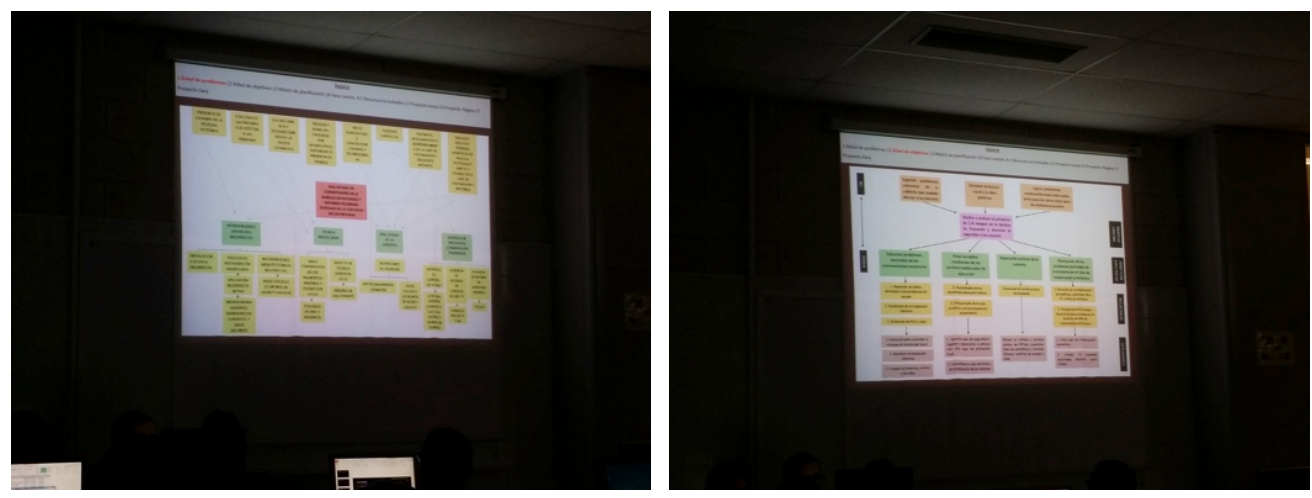

Fig. 14 y 15. Exposición de dos Trabajos finales de la Asignatura Desarrollo, gestión y dirección de proyectos de restauración, curso 2015-2016. Máster Universitario en Conservación y Restauración de Bienes Culturales, del Departamento de Conservación y Restauración de Bienes Culturales de la UPV. 
Por otro lado, esta técnica puede utilizarse en otros campos como el de la Gestión, en el diseño de un servicio y en el de un nuevo modelo de negocio. El método permitiría contrastar diferentes alternativas en ambos casos, dando lugar a un mayor número de ideas.

Por último, se podría plantear un análisis cuantitativo de los modelos creados utilizando la metodología del análisis de redes (social network analysis) para evaluar la estructura de cada modelo (De Miguel y Herrero, 2012). Podríamos tratar las herramientas (nodos) como actores para medir a) la densidad y b) la centralización del proyecto. La densidad se calcula dividiendo el actual número de conexiones entre el máximo número posible. A mayor densidad, mayor cohesión y mejor intercambio de información. La centralización se calcula midiendo primero el nivel de centralización de cada nodo en relación al más centralizado, para después dividirlo entre el mayor número posible de diferencias. La centralización de una red puede aportar integración. De este modo, cuanto más conectado está un nodo (en número de relaciones directas) su centralidad será mayor (diríamos que será una herramienta clave). Si la red es demasiado densa, será menos innovadora, mientras que si es demasiado fluida, su eficacia disminuirá. Por tanto podemos decir que lo mejor sería un equilibrio que permitiera cierto nivel de conflicto para fomentar la innovación, a la vez que algún grado de cohesión que permita alcanzar la eficacia necesaria.

\section{Referencias}

BUZÁN, T. (1996). The Mind Map Book: How to Use Radiant Thinking to Maximize Your Brain's untapped potential. New York (USA): Plume.

DE Miguel MOLINA, M. Y HeRrero BlASCO, A. (2012). Propuesta de uso del análisis de redes de actores de políticas públicas para la gestión de la integración de los Menores Inmigrantes No Acompañados (MINA). Estudio del caso de la Comunidad Valenciana. Revista sobre la infancia y la adolescencia, n. 3, p. 33-45. doi:http://dx.doi.org/10.4995/reinad.2012.1131.

FERRERO Y DE LOMA-OSORIO, G. Ed. (2009). Identificación y formulación de proyectos de cooperación para el desarrollo: gestión del ciclo del proyecto y enfoque del marco lógico. Valencia: Editorial UPV.

GARCÍA CANCLINI, N. Ed. (1993). El consumo cultural en México. México: Consejo Nacional para la Cultura y las Artes.

MUÑOZ GONZÁLEZ, J. M., SERRANO RODRÍGUEZ, R., MARÍN DÍAZ, V. (2015). El aprendizaje colaborativo y su desarrollo a través de mapas mentales. Una innovación educativa en la formación inicial docente. Educatio Siglo XXI, Vol.32.

WUJEC, T (2013). Got a wicked problem? First, tell me how you make toast. TED Global 2013.

$<$ https://www.ted.com/talks/tom wujec got a wicked problem first tell me how you make toast?language $=$ en $>$ [Consulta: 22 de marzo de 2016].

(c) EY-NC-ND 2016, Universitat Politècnica de València 


\section{Agradecimientos :}

Agradecer a las alumnas Andrea Cantos Guillen, Rocio Castellote Simon, Elisa Martinez Zeron y Marina Ortiz Gonzalez de la tercera promoción del Grado en Conservación y Restauración de Bienes Culturales, del Departamento de Conservación y Restauración de Bienes Culturales de la UPV, por su apoyo y participación en una de las dinamicas expérimental. 